\title{
Clinical Manifestations and Diagnostic Challenges of Tinea faciei
}

\author{
Kushwaha Pragya, Thakur Rameshwari, Kumar Harish and Avneet Singh Kalsi* \\ Department of Dermatology and Microbiology, Muzaffarnagar Medical College, Opp. \\ Beghrajpur Industrial Area, Meerut Road, Muzaffarnagar, U.P., India \\ *Corresponding author
}

\begin{tabular}{|c|c|}
\hline & 7 \\
\hline & \multirow{6}{*}{$\begin{array}{l}\text { Dermatophyte infections are common superficial fungal infections and are prevalent all } \\
\text { over the world. Some dermatophytes are cosmopolitan in distribution, while others are } \\
\text { geographically restricted. In an immunocompetent host, the lesions have typical } \\
\text { appearance of being annular and scaly with central clearing. But, in patients with } \\
\text { HIV/AIDS or any other immunosuppression, the lesions can be extensive and without } \\
\text { central clearing. Recently, the Indian scenario has changed due to inadvertent use of } \\
\text { topical steroids and we often come across atypical and extensive lesions without central } \\
\text { clearing. Due to sudden rise in the incidence of tinea faciei for the past few years, a study } \\
\text { was conducted in detail in the current clinical pattern and mode of transmission of tinea } \\
\text { faciei and to isolate the dermatophyte associated with it. Patients with typical and atypical } \\
\text { dermatophytic lesions and KOH and/or culture positive were included in the study. } \\
\text { Samples were collected from the affected area after cleaning the part with } 70 \% \text { ethyl } \\
\text { alcohol. Samples were planted on Sabouraud Dextrose Agar (SDA), supplemented with } \\
\text { chloramphenicol and cycloheximide. The commonest clinical pattern of tine faciei in } \\
\text { males was ill-defined scaly lesions without signs of inflammation } 15(35.71 \%) \text { and in } \\
\text { females, erythematous plaques with pustules and without central clearing, was the } \\
\text { commonest lesion } 16(44.44 \%) \text {. None of the patient had any immunosuppression except } \\
\text { few had diabetes mellitus. Out of a total } 78 \text { samples, } 57 \text { ( } 73.07 \%) \text { were Trichophyton } \\
\text { interdigitale, one case (1.28\%) was due to Trichophyton violaceum and no other species } \\
\text { were found. Nearly } 30 \% \text { of the patients couldn't recollect the name of the topical cream } \\
\text { used by them. Molecular typing of the isolates was not done. Tinea faciei should be } \\
\text { considered as a separate clinical entity. Some of the facial lesions can mimic other clinical } \\
\text { conditions and we are coming across more cases of tinea faciei as compared to reported in } \\
\text { the past. Awareness among the patients has to be created about taking appropriate } \\
\text { treatment from the dermatologists; otherwise it may lead to an epidemic, difficult to } \\
\text { control. }\end{array}$} \\
\hline & \\
\hline $\begin{array}{l}\text { Tinea faciei, } \\
\text { Trichophyton } \\
\text { interdigitale, } \\
\text { Trichophyton } \\
\text { violaceum. }\end{array}$ & \\
\hline Article & \\
\hline & \\
\hline & \\
\hline
\end{tabular}

\section{Introduction}

Tinea infections are considered among the most common dermatologic conditions all over the world. India being a tropical country, many people are affected. Though it has been found to have perennial presentation, but the spikes are seen during rainy season.
Studies on the prevalence of dermatophytes have been carried out in different regions of India from time to time, and Trichophyton rubrum has been found to be the predominant species in most of the regions. Some of the studies have been listed in Table 1 . Here in a 
tertiary care centre in Western U.P., Trichophyton interdigitale (formerly Trichophyton mentagrophyte), has been found to be the predominant dermatophyte species.

According to a study carried out here recently, Trichophyton interdigitale was the commonest dermatophyte species isolated $98.5 \%$, Trichophyton rubrum $0.5 \%$ and Trichophyton violaceum $1.28 \%$ (unpublished data).

The causative agent of dermatophyte can belong to any of the three genera: Trichophyton, Epidermophyton or Microsporum, and their species, which have specific geographical distribution.

According to the different body sites involved, the clinical diagnosis can be named as tinea faciei, tinea corporis, tinea genitalis, tinea cruris, tinea capitis, tines pedis and tinea unguium. Among these, tinea corporis is the commonest clinical type, followed by tinea cruris.

Due to unethical use of topical steroids, the lesion can be extensive, atypical and without central clearing. The clinical form, tinea faciei, once thought to be uncommon, is now being reported with increased frequency. Keeping this in mind, we conducted a study on the clinical profile of tinea faciei and its mycological aspect and mode of transmission.

We noticed five clinical pattern of tinea faciei according to its typical morphological features, which are given below:

Classical annular pattern with raised scaly margins and central clearing (Figure 1).

Circular erythematous plaques, studded with pustules and without central clearing (most common finding in children and females) (Figure 2).
Annular or serpiginous border only encircling either whole of the face or covering forehead and temple area (most common presentation in adult males) (Figure 3).

Erythematous plaques unilaterally or bilaterally distributed on the malar area of face (Figure 4).

Ill-defined scaly lesions anywhere on the face, without the signs of inflammation (Figure 5).

\section{Materials and Methods}

The study was conducted at a tertiary care centre of Western U.P. in North India from July 2015 to June 2016. Permission to conduct the study was taken from the ethical committee of the institute.

\section{Inclusion criterion}

Clinically typical or clinically suspicious (atypical forms), but $\mathrm{KOH}$ positive and/or culture positive cases of tinea faciei of all ages and both sexes were included in the study

Data was recorded in the form of age, sex, site on the face, duration, clinical presentation, treatment taken, dermatophyte species identification and the probable source of contact and mode of acquiring the disease.

Non-bearded area of adult males were considered as tinea faciei and so included in the study.

Patients were sent to the Microbiology department of a tertiary care centre for the collection and processing of the samples.

\section{Methods}

The sample was collected after thoroughly scrubbing the suspicious area with $70 \%$ ethyl alcohol. The scales were collected from the 
periphery with the help of sterile scalpel blade in a sterile petri dish. Skin scrapings or nail clippings were collected from other sites, if present and were processed separately. All the samples were examined for fungal elements in Potassium hydroxide $(\mathrm{KOH}) 20 \%$ mount under high power of the microscope. Both positive and negative samples were inoculated on Sabouraud Cycloheximide Chloramphenicol Agar (HiMedia). The plates were incubated at $25^{\circ} \mathrm{C}$ for a period of four weeks and were observed every week for growth.

Culture positive plates were observed for colony characteristics both on surface and reverse. LactoPhenol Cotton Blue (LPCB) preparations by teased mount method and scotch tape method were prepared to study the microscopic structures in detail. Urease test and in vitro hair perforation tests were performed for the confirmation of Trichophyton interdigitale species. Also, other standard tests needed for the identification of dermatophytes were performed according to the description given in various textbooks, manuals, and journals.

\section{Results and Discussion}

A total number of 78 patients were enrolled for the study, out of which $48(62.50 \%)$ patients were males and $30(37.5 \%)$ were females.

Annular lesions without central clearing and pustules, was the most common presentation in $20(25.64 \%)$ patients (Figure 2), followed by plaque like lesions in $16(20.51 \%)$ patients (Figure 4). Ill-defined scaly lesions without signs of inflammation were seen in 15 (19.23\%) patients (Figure 5), classical annular lesions were seen in $14(17.95 \%)$ patients (Figure 1) and only raised serpiginous border without papules were present in 13 (16.67\%) patients (Figure 3).
Most common age group was between 21-30 years (Table 2). Lowest age in males was one year and in females was three years.

The most common site on the face was cheek in children and females and forehead and temple area in males.

Atypical forms, which were found to be both $\mathrm{KOH}$ and culture negative, were investigated further for the diagnosis of rosacea, seborrheic dermatitis, contact dermatitis, and lupus erythematosus by histopathological studies. Such atypical cases were not included in the study. Associated findings besides scaling and erythema were burning, itching and photosensitivity. None of them had vesicular presentation. Site of presentation was cheek, forehead, temple and chin in descending order, bilateral malar eminences, periorbital and ear area were also involved in some.

Body sites other than face were affected in 42 patients mainly as tinea corporis or tinea cruris. Tinea unguium of fingernails were seen in two patients, and tinea capitis was not seen in any child with tinea faciei.

History of topical steroid application was found in patients with atypical clinical forms of tinea faciei (Figure 2-5).

There were few patients of tinea incognito of face with clinical pictures of hypopigmented scaly plaques on nose, right cheek extending upto ear (Figure 6), 'Ring-within-a-ring' over right cheek (Figure 7) and 'Double edged tinea' over right cheek (Figure 8). They were all included in clinical pattern group 5.

\section{Mycological features}

Among 78 cases of tinea faciei, all were $\mathrm{KOH}$ positive, 58 (74.36\%) were culture positive. On KOH examination, branching septate 
hyphae were seen. $T$. interdigitale was isolated in 57 cases and $T$. violaceum was found only in one case and other species were not found. Colonies of $T$. interdigitale were powdery to fluffy, cream to white in obverse and yellow to brown on reverse (Figure 9). Lacto Phenol Cotton Blue mounts were prepared, which showed septate fungal hyphae, with numerous spherical microconidia arranged in grape-like clusters, cigar shaped macroconidia and spiral hyphae (Figure 10). Hot and humid climate of western Uttar Pradesh along with topical steroid abuse is possibly responsible for such a large number of dermatophyte infections. One third of daily OPD attendance consists of dermatophyte infection. Extensive and atypical tinea corporis with no central clearing is the most common clinical presentation, followed by tinea cruris, tinea genitalis, tinea unguium, tinea capitis and tinea pedis in descending order. Tinea faciei can pose a diagnostic problem, being on exposed area and application of cosmetics and steroid creams can mask some of the clinical features.
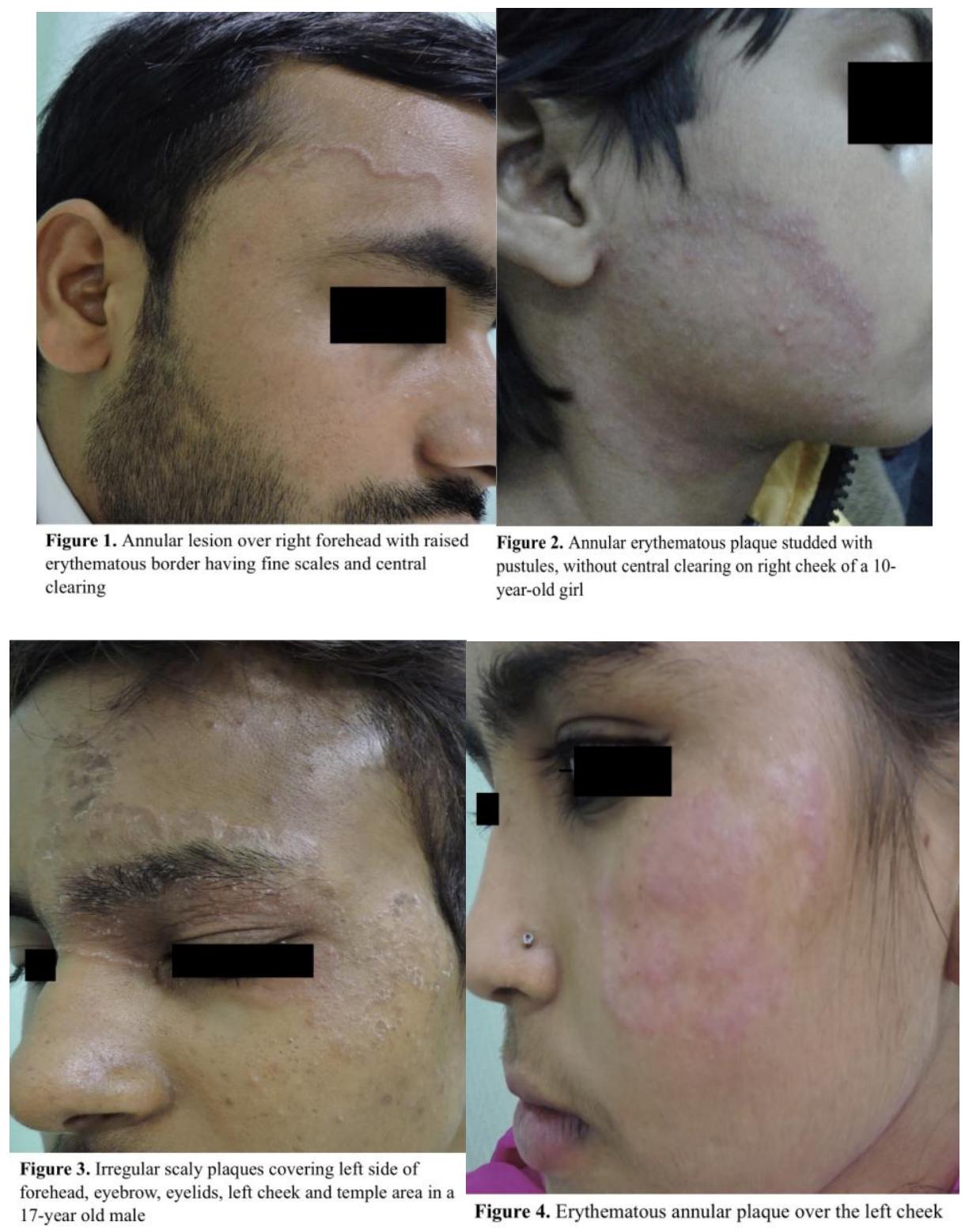


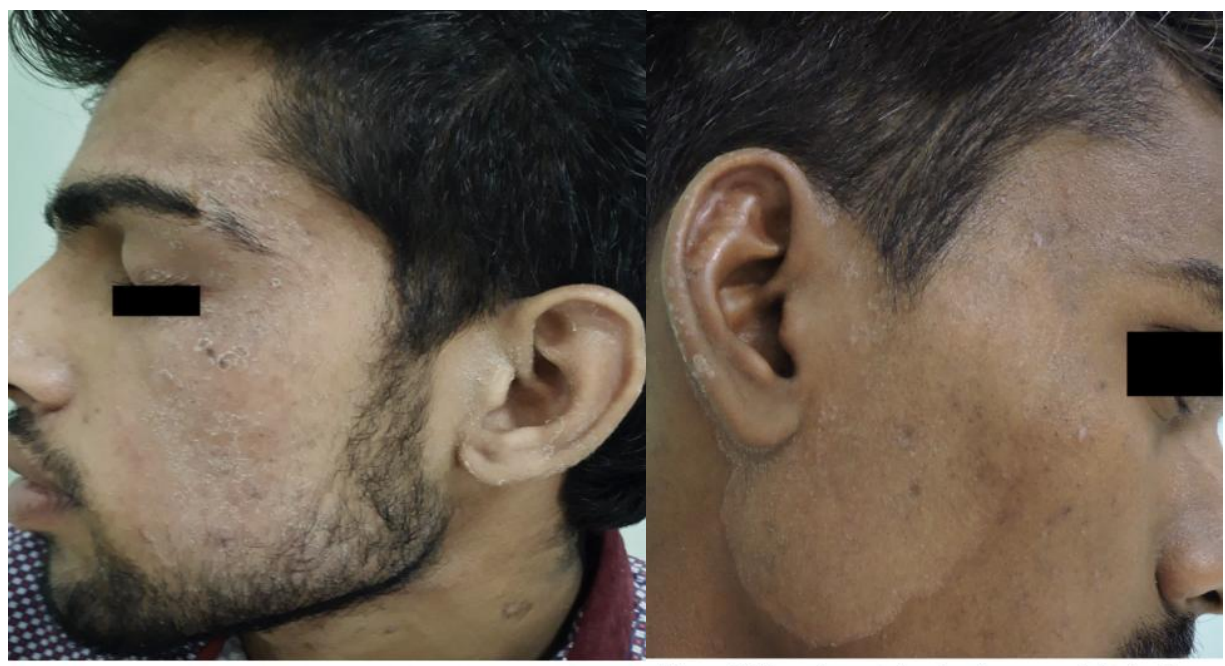

Figure 5. Irregular scaly plaque with involvement of ear

Figure 6. Hypopigmented scaly plaques on right cheek, and left side of face 'ear sign' nose and right ear

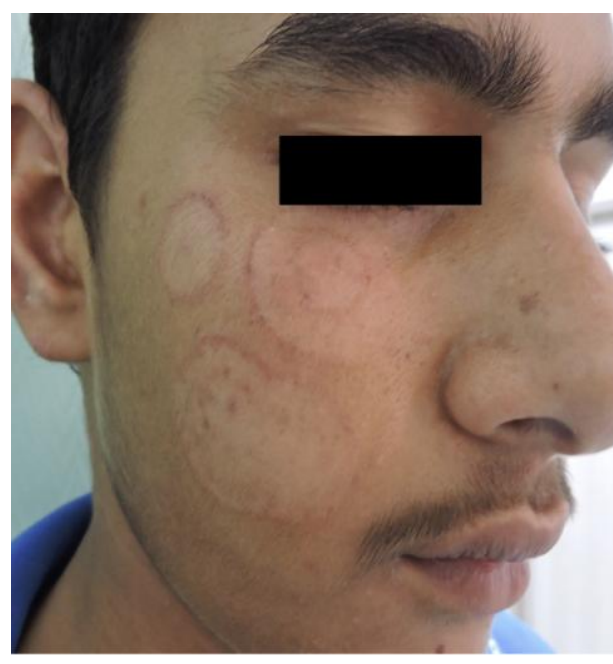

Figure 7. Three annular hypopigmented lesion on right cheek. The one below eye has 'ring-within-a-ring' appearance

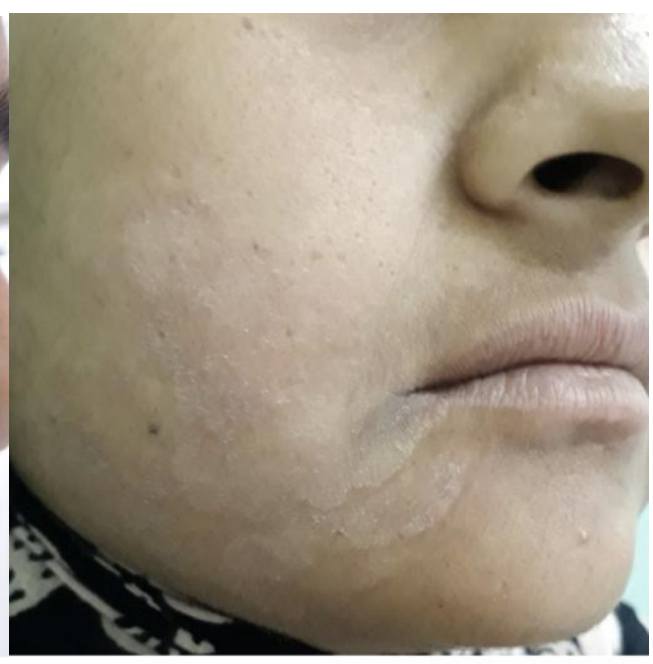

Figure 8. Ill-defined hypopigmented plaques on right cheek with 'double-edged' tinea due to use of topical steroid

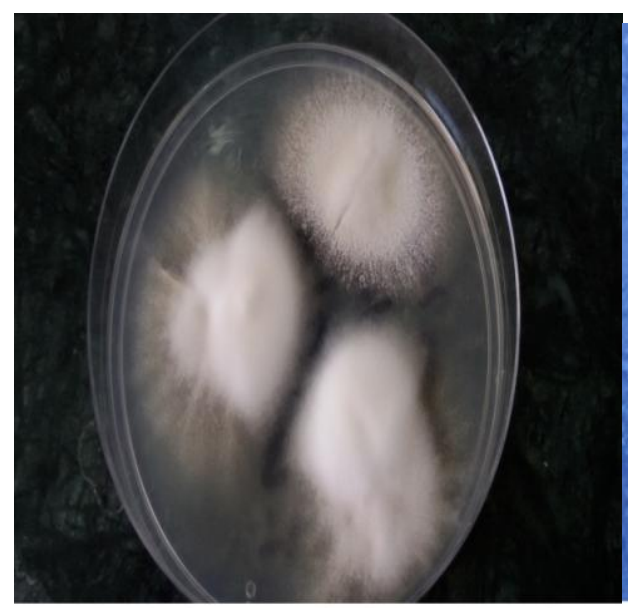

Figure 9. Mixed appearance of colonies; colony on the top with granular appearance and other two having cottony appearance

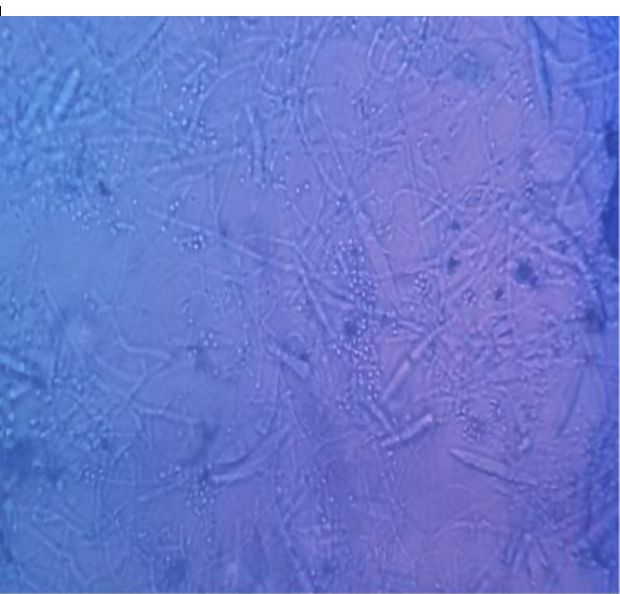

Figure 10. LCB mount $T$. interdigitale showing both cigar shaped macroconidia and microconidia in grape like clusters and some spiral hyphae 
Table.1 Results of various studies in India on the prevalence of dermatophytes

\begin{tabular}{|c|c|c|c|c|}
\hline Author & Place of Study & $\begin{array}{l}\text { Predominant } \\
\text { species }\end{array}$ & $\begin{array}{l}\text { Second Predominant } \\
\text { species }\end{array}$ & $\begin{array}{l}\text { Other } \\
\text { dermatophytes }\end{array}$ \\
\hline $\begin{array}{l}\text { Noronha } \text { et al. } \\
2016\end{array}$ & North Karnataka & $\begin{array}{l}T . \\
\text { mentagrophyte } \\
48.3 \%\end{array}$ & T. rubrum $38.3 \%$ & T. violaceum $5 \%$ \\
\hline Poluri LV.2015 & Telangana & $\begin{array}{l}\text { T. rubrum } \\
58.06 \%\end{array}$ & $\begin{array}{l}T . \\
\text { mentagrophyte } 22.58 \%\end{array}$ & \\
\hline $\begin{array}{l}\text { Lakshmanan et } \\
\text { al., } 2015\end{array}$ & Tamil Nadu & T. rubrum $79 \%$ & $\begin{array}{l}\text { T. mentagrophyte } \\
14.5 \%\end{array}$ & \\
\hline $\begin{array}{l}\text { Surendran et al., } \\
2014\end{array}$ & Mangalore & $\begin{array}{l}\text { T. rubrum } \\
67.5 \%\end{array}$ & T. mentagrophyte $20 \%$ & \\
\hline $\begin{array}{l}\text { Bhatia VK, } \\
\text { Sharma PC. } \\
2014\end{array}$ & $\begin{array}{l}\text { Himachal } \\
\text { Pradesh }\end{array}$ & $\begin{array}{l}T . \\
\text { mentagrophyte } \\
63.5 \%\end{array}$ & T. rubrum $35.1 \%$ & $\begin{array}{l}\text { M. canis } 3.2 \% \\
\text { M. gypseum } \\
3.2 \%\end{array}$ \\
\hline $\begin{array}{l}\text { Sumana } \\
\text { et al., } \\
\mathbf{2 0 0 4}\end{array}$ & $\begin{array}{l}\text { Khammam } \\
\text { Andhra Pradesh }\end{array}$ & T. rubrum $60 \%$ & T. violaceum $26 \%$ & \\
\hline $\begin{array}{l}\text { Grover \& Roy. } \\
\mathbf{2 0 0 3}\end{array}$ & North-East India & $\begin{array}{l}\text { T. tonsurans } \\
20.5 \%\end{array}$ & T. rubrum 9\% & $\begin{array}{l}\text { M. ferrugineum } \\
5.8 \% \mathrm{~T} \text {. } \\
\text { mentagrophyte } \\
2.9 \%\end{array}$ \\
\hline $\begin{array}{l}\text { Parwardhan } e t \\
\text { al.,1999 }\end{array}$ & $\begin{array}{l}\text { Aurangabad } \\
\text { Maharashtra }\end{array}$ & $\begin{array}{l}\text { T. rubrum } \\
28.12 \%\end{array}$ & $\begin{array}{l}\text { T. mentagrophyte } \\
25.0 \%\end{array}$ & \\
\hline $\begin{array}{l}\text { Karmakar et al., } \\
1995\end{array}$ & $\begin{array}{l}\text { Western } \\
\text { Rajasthan }\end{array}$ & $\begin{array}{l}\text { T. violaceum } \\
55.76 \%\end{array}$ & T. rubrum $42.3 \%$ & \\
\hline $\begin{array}{l}\text { Gupta et al., } \\
\mathbf{1 9 9 3}\end{array}$ & Ludhiana & $\begin{array}{l}\text { T. rubrum } \\
42.42 \%\end{array}$ & E. flocossum $15.15 \%$ & $\begin{array}{l}T . \\
\text { mentagrophyte } \\
6.06 \%\end{array}$ \\
\hline
\end{tabular}

Table.2 Age wise distribution of Tinea faciei

\begin{tabular}{|c|c|c|}
\hline Age & Male & Female \\
\hline $0-10$ & $2(4.76 \%)$ & $4(11.11 \%)$ \\
\hline $11-20$ & $14(33.33 \%)$ & $6(16.67 \%)$ \\
\hline $21-30$ & $20(47.62 \%)$ & $18(50.00 \%)$ \\
\hline $31-40$ & $4(9.52 \%)$ & $7(19.44 \%)$ \\
\hline $41-50$ & $2(4.76 \%)$ & $1(2.78 \%)$ \\
\hline TOTAL & 42 & 36 \\
\hline
\end{tabular}


Table.3 Clinical pattern of tinea faciei

\begin{tabular}{|l|l|l|l|}
\hline & Clinical pattern & Male & Female \\
\hline 1 & Classical annular & $6(14.29 \%)$ & $8(22.22 \%)$ \\
\hline 2 & Erythematous plaques and pustules without central clearing & $4(9.25 \%)$ & $16(44.44 \%$ \\
\hline 3 & Serpiginous border like & $13(30.95 \%)$ & - \\
\hline 4 & Granulomatous plaques either U/L or B/L on malar eminences & $4(9.525 \%)$ & $12(33.33 \%$ \\
\hline 5 & Ill-defined scaly lesions without signs of inflammation & $15(35.71 \%)$ & \\
\hline & Total & $42(100 \%)$ & $36(100 \%)$ \\
\hline$\chi^{2}$ Chi Square) value= 39.256 at DF4. P-value <0.0001 (Highly Significant) \\
\hline
\end{tabular}

As depicted in Table 3, tinea faciei patients with classical annular lesions with raised scaly margins with central clearing, were not using topical or systemic steroids for the treatment, so can be categorized as typical forms but in later four categories, patients gave history of using steroids either in the form of injections, tablets or creams and were placed in atypical forms. So in these cases, clinical picture of tinea was lost and diagnosis was made after thorough history, clinical examination, $\mathrm{KOH}$ mount and culture.

Tinea faciei, unlike other types of tinea, has not gained much attention and has been often treated as part of tinea corporis. But according to a study 'A 20 -year survey of tinea faciei' by Nicola et al., it has been strongly recommended that tinea faciei should be considered as separate entity and should be differentiated from other inflammatory facial dermatitis. According to various authors, it has been recommended that tinea faciei should be considered as distinct clinical form because of the facial anatomy and physiological characteristics, frequent washing, exposure to sun and application of cosmetics can lead to atypical clinical presentation, which may result in incorrect diagnosis.

Most of those adult male patients gave history of having visited salons for haircut and facial massage. In majority of children, history of tinea was positive in their family members. In cases of tinea corporis and tinea cruris, there can be involvement of face due to inoculation. But, in patients with exclusive tinea faciei, we could not get the exact source of infection.

Some of the outstanding and extensive studies carried out recently by Verma and Madhu (2017), who has great expertise in dermatophytosis, has mentioned about the inadvertent and unethical use of topical steroids, due to which dermatophytoses has acquired epidemic proportion and also tinea faciei is being reported with increased frequency. The scenario here in Western U.P. fits into his description except in this region, T. interdigitale predominates.

All the isolates of $T$. interdigitale from tinea faciei phenotypically appeared like anthropophilic strains and clinically also the lesions looked like due to anthropophilic strain. They were not inflammatory or ulcerative like in case of zoophilic infections. The anthropophilic species usually cause more chronic, less circumscribed infections, which result in less resistance to re-infection.

The colonies of anthropophilic strains are cottony in appearance and that of zoophilic strains have granular appearance. At times, the colony may have mixed appearance (Figure 9).

Limitation of the study is that we did not do the molecular typing of the isolates. It is 
usually not possible to differentiate between the anthropophilic strains and zoophilic strains of $T$. interdigitale on morphological and biochemical basis alone.

Though, clinically, it is also seen that infection due to zoophilic strains is acute and inflammatory and at times, may heal with scarring. Infection due to anthropophilic strain is chronic and contagious.

But, molecular identification methods are needed for the differentiation of zoophilic strains of T. interdigitale, T. mentagrophyte, and Trichophyton anamorph of Arthroderma benhamiae.

Tinea faciei is a clinical condition which needs our special attention due to diagnostic challenges associated with it. At times, patient with tinea faciei may complain about the facial lesion, which can be just the tip of the iceberg and without mentioning about tinea cruris/genitalis and the patient could have acquired tinea faciei due to autoinoculation. Sample should be collected and processed from different lesions present in a patient.

There is rising incidence of tinea faciei recently. Multiple factors could be responsible like frequent visits to beauty parlours, and salons for facial massage in case of exclusive tinea faciei. Unethical mixing of potent topical steroids in cosmetics such as fairness cream when used by a patient of tinea faciei can worsen the condition and can lead to atypical clinical presentation (steroid modified tinea) along with adverse effects which are considered to be more on face and can result in tinea faciei incognito.

Inadvertent use of topical steroids and relative lack of local cutaneous immunity are probable factors for persistent nature of the disease, results in extensive and atypical lesions and delay the appropriate treatment.

\section{References}

Aste N, Pau M, Biggio P. Micosi atipicheNota I: tinea faciei. Rass Med Sarda 1978; 91:3-8.

Bhatia and Sharma: Epidemiological studies on Dermatophytosis in human patients in Himachal Pradesh, India. Springer Plus 2014; 3:134.

Grover S, Roy P. Clinico-mycological Profile of Superficial Mycosis in Northeast India. MJAFI 2003 Vol 59 No.2 11416.

Gupta A K, Choudhary M, Elewski B. Tinea corporis, tinea cruris, tinea nigra, and piedra. Dermatol Clin 2003; 395-400.

Gupta BK, Kumar S, Kumar RA, Khurana S. Mycological aspects of dermatomycoses in Ludhiana. Indian $\mathbf{J}$ Pathol Microbiol 1993; 36(3): 233-37.

Karmarkar S, Kalla G, Joshi KR, Karmarkar S. Dermatophytoses in a desert district of Western Rajasthan. India J Dermatol Venereol Leprol 1995; 61:280-83.

Lahiri K, Coondoo A. Topical steroid damaged/ dependent face (TSDF): An entity of cutaneous pharmaco dependence. Indian J Dermatol 2016; 61:265-72.

Lakshmanan A, Ganeshkumar P, Mohan SR, Hemamalini M, Madhavan R. Epidemiological and clinical pattern of dermatomycoses in rural India. Indian $\mathbf{J}$ Med Microbiol 2015; 33:S134-36.

Lin RL, Szepietowski JC, Schwartz RA. Tinea faciei: an often deceptive facial eruption. Int J Dermatol 2004; 42:43740.

Nenoff P, Herrmann J, Gräser Y. Trichophyton mentagrophtes sive interdigitale? A dermatophyte in the course of time. Dtsch Dermatol Ges 2007; 5:198-202.

Nicola A, Laura A, Natalia A, Monica P. A 20-year survey of tinea faciei. Mycoses 2010; (6): 504-8. 
Noronha TM, Tophakhane RS, Nadiger S. Clinico-mycological study of dermatomycoses in a tertiary-care hospital in North Karnataka. Indian Dermatol online J 2016; 7:264-71.

Sumana V, Singaracharya MA. Dermatophytosis in Khammam (Khammam district, Andhra Pradesh, India). Indian J Pathol Microbiol 2004 April, 47(2): 287-89.

Patwardhan N, Dave R. Dermatomycoses in and around Aurangabad. Indian J Pathol Microbiol 1999; 42(4): 455-62.

Poluri LV, Indugula JP, Kondapaneni SL. Clinicomycological study of dermatophytosis in South India. J Lab Physicians 2015; 7:84-89.

Romano C, Ghilardi A, Masaai L. Eighty-four consecutive cases of tinea faciei in Siena, a retrospective study (19892003). Mycoses 2005; 48:343-6.

Surendran KAK, Bhat RM, Boloor R, Nandkishore B, Sukumar D. A Clinical and mycological Study on Dermatophyte Infections. Indian $\mathbf{J}$ Dermatol 2014 May-Jun; 59(3): 262-67. Verma SB, Madhu R. The great Indian Epidemic of superficial dermatophytosis: An appraisal. Indian J Dermatol 2017; 62:227-36.

\section{How to cite this article:}

Kushwaha Pragya, Thakur Rameshwari, Kumar Harish and Avneet Singh Kalsi. 2017. Clinical Manifestations and Diagnostic Challenges of Tinea faciei. Int.J.Curr.Microbiol.App.Sci. 6(12): 1286-1294. doi: https://doi.org/10.20546/ijcmas.2017.612.145 